\title{
Epstein-Barr Virus Survival: Expression and Release of Fas Ligand
}

\begin{abstract}
Key words: FasL, LMP1, LYDMA, cytotoxic T cell, NK cell, RCAS1
\end{abstract}

\section{EBV and Clinical Diseases}

Epstein-Barr virus (EBV) belongs to the herpesvirus group and is associated with a wide spectrum of clinical diseases. Primary EBV infection usually occurs within the first 3 years of life and is almost always asymptomatic (1). By contrast, primary infection in Western countries is often delayed until the second decade or later, and induces infectious mononucleosis, which may rarely be complicated with hemophagocytic syndrome (1). EBV also rarely induces persistently active infection, namely, chronic active EBV infection, in which chronic activation of EBV persists, presenting various clinical symptoms, including immunodeficiency, hepatic and hematological disorders, and often leads to the development of malignant transformation of EBV infected cells $(2,3)$. In addition, EBV has been reported to be associated with many malignant diseases, not only Burkitt lymphoma, but nasopharyngeal carcinoma, Hodgkin's disease, gastric cancer, nasal NK/T cell lymphoma, malignant histiocytosis, and leiomyosarcoma (1-4).

\section{EBV and Host Immune Responses}

Why is EBV associated with this wide range of clinical diseases including malignant neoplasms? It is related to the specific features of EBV itself and the host immune responses to control EBV infection. EBV initially infects oropharyngeal epithelium and/or B cells, where propagation and complete maturation of $\mathrm{EBV}$ occur. In addition, the oropharynx provides the main site for the persistent and latent infection of EBV in healthy individuals after recovery from primary EBV infection (1, 2). EBV also infects B cells, and sometimes other lymphocytes, via CD21 (C3d receptor) and transforms B cells, expressing LYDMA, which mainly consists of latent membrane protein (LMP) and EBNA2 $(1,2)$. Interestingly, LMP1 was shown to stimulate tumor necrosis factor receptor associated factor (TRAF) which mediates proliferative signals, and leads to the transformation of the EBV infected cells (5). Since the LYDMA is a target antigen of reactive $\mathrm{CD} 8$ positive cytotoxic $T$ cells, transformation of EBV infected cells by LMP1 conversely induce effective elimination of those cells by the immune system (2). Thus, in healthy people, activation of EBV at oropharynx and elimination of EBV virions and/or EBV transformed cells by host immune responses is ingeniously balanced.

\section{Strategies of EBV for Survival}

On the other hand, EBV also has many mechanisms to support the viability of EBV infected cells and also those to evade from immunologic attacks of the host. EBV encodes the BHRF1 gene, which has functional homology to Bcl-2 that may operate to increase the survival of the EBV infected cells (1). Moreover, BCRF1 gene of EBV (vIL-10), which has extensive homology to human IL-10 gene, may contribute to shift the host immune response from Th1 to Th2 type immune response (13). Since EBV infected cells are eliminated by CD8 positive cytotoxic T cell responsiveness, depending on the Th1 type immune response, to deviate the immune response from Th1 to Th2 may contribute to increase the possible survival of EBV infected cells. In addition, production of vIL-10 may play some role in the allergic reaction, such as hypersensitivity against mosquito bites observed in a patient with chronic EBV infection (6).

\section{See also $\mathrm{p} 651$.}

\section{FasL: Another Escape Mechanism from the Immune System of EBV}

Furthermore, recently, accumulating evidence including the clinical report which appears in this issue, suggested that EBV infected cells express and release Fas ligand (FasL) $(6,7)$. Activated $\mathrm{T}$ cells express Fas on their surfaces, and will be killed by FasL, of which mechanism prevents the overshoot of immune response (8). The production of FasL in EBV infected and/or transformed cells may contribute to the increased survival of EBV transformed cells evading from host immune responses, leading to the development of malignant neoplasms. However, recently, it was reported that RCAS1 (receptor-binding cancer antigen expressed on SiSo cells), which is a new apoptosis inducing factor against immune cells, rather than FasL was expressed in Reed-Sternberg cells in Hodgkin's disease in association with apoptotic lymphocytes surrounding ReedSternberg cells $(9,10)$. Thus, immune escape strategies of EBV infected cells may be more complex, consisting of multiple molecules and many mechanisms. 
Further studies are necessary to clarify the escape mechanisms from host immune system in EBV transformed cells, and those studies will contribute to the possible prevention and control of the development of EBV associated malignant diseases.

Seiho Nagafuchi, MD, PhD Department of Medical Technology, School of Health Sciences, Kyushu University, Fukuoka 812-8582

\section{References}

1) Kieff E. Epstein-Barr virus and its replication. in: Fields' Virology. 4th ed. Knipe DM, Howley PM, et al, Eds. Philadelphia, Pennsylvania, Lippincott-Raven, 2001: 2575-2627.

2) Nagafuchi S. Epstein-Barr virus and carcinogenesis. Curr Conc Inf Dis 19:12-15, 2000 (in Japanese).

3) Nagafuchi S, Ohtsuka T, Niho Y. Chronic EB virus infection and cytokine: IL-2, interferon- $\gamma$, and BCRF1 gene product (Viral IL-10). Intern Med 32: 945-947, 1993.

4) Fujisaki T, Nagafuchi S, Niho Y, Tsuneyoshi M, Oshima K, Kikuchi M.
Malignant histiocytosis in a patient with chronic active Epstein-Barr virus infection. Lancet 340: 1168-1169, 1992.

5) Mosialos G, Birkenbach M, Yalamanchili R, VanArsdale T, Ware C, Kieff E. The Epstein-Barr virus transforming protein LMP1 engages signaling proteins for the tumor necrosis factor receptor family. Cell 80: 389-399, 1995.

6) Ohshima $\mathrm{S}$, Ishii M, Asada $\mathrm{H}$, et al. A possible mechanism of NK celllineage granular lymphocyte proliferative disorder (NK-GLPD) in a patient with chronic active Epstein-Barr virus infection (CAEBV) and severe hypersensitivity to mosquito bites (SHMB). Intern Med 41: 651656, 2002.

7) Ohshima K, Suzumiya J, Sugihara M, Nagafuchi S, Ohga S, Kikuchi M. CD95 (Fas) ligand expression of Epstein-Barr virus (EBV)-infected lymphocytes: a possible mechanism of immune evasion in chronic active EBV infection. Pathol Int 49: 9-13, 1999.

8) Nagata S, Golstein P. The Fas death factor. Science 267: 1449-1456, 1995.

9) Nakashima M, Sonoda K, Watanabe T. Inhibition of cell growth and induction of apoptotic cell death by the human tumor-associated antigen RCAS1. Nat Med 5: 938-942, 1999.

10) Ohshima K, Muta K, Nakashima M, et al. Expression of human tumorassociated antigen RCAS1 in Reed-Sternberg cells in association with Epstein-Barr virus infection: A potential mechanism of immune evasion. Int J Cancer 93: 91-96, 2001. 